\title{
RNA chaperones encoded by RNA viruses
}

\author{
Jie Yang, Hongjie Xia, Qi Qian, Xi Zhou
}

State Key Laboratory of Virology, College of Life Sciences, Wuhan University, Wuhan 430072, China

RNAs are functionally diverse macromolecules whose proper functions rely strictly upon their correct tertiary structures. However, because of their high structural flexibility, correct folding of RNAs is challenging and slow. Therefore, cells and viruses encode a variety of RNA remodeling proteins, including helicases and RNA chaperones. In RNA viruses, these proteins are believed to play pivotal roles in all the processes involving viral RNAs during the life cycle. RNA helicases have been studied extensively for decades, whereas RNA chaperones, particularly virus-encoded RNA chaperones, are often overlooked. This review describes the activities of RNA chaperones encoded by RNA viruses, particularly the ones identified and characterized in recent years, and the functions of these proteins in different steps of viral life cycles, and presents an overview of this unique group of proteins.

\section{KEYWORDS RNA viruses; RNA chaperone; ATP-independent helix-destabilizing activity; kinetic trap; viral life cycle}

\section{INTRODUCTION}

Most RNA molecules rely on correct tertiary structures for their proper functions. However, owing to their extreme structural flexibility, correct folding of RNAs appears to be a difficult process. RNAs can easily become kinetically trapped in alternative secondary and tertiary structures that are misfolded and inactive. However, because these alternative misfolded structures of RNAs contain a multitude of base-pairings and duplexes that are locally stable, they are very hard to refold. Such a state of RNA misfolding is named a "kinetic trap" (Chauhan and Woodson, 2008).

To deal with this problem, hosts and viruses encode "helper" or RNA remodeling proteins, such as ATP-independent RNA chaperones and ATP-dependent RNA helicases. These proteins can destabilize RNA-RNA or RNA-DNA base-pairings, lowering the thermodynamic barrier between RNA conformations to help RNA es-

Received: 5 November 2015, Accepted: 11 December 2015,

Published Online: 22 December 2015

$\triangle$ Correspondence:

Phone: +86-27-68756654, Fax: +86-27-68754941,

Email: zhouxi@whu.edu.cn

ORCID: 0000-0002-3846-5079 cape from kinetic traps and refold to form proper RNA structures (Chauhan and Woodson, 2008). RNA remodeling proteins play critical roles in almost all processes of RNA metabolism.

RNA remodeling proteins are comprised of two large, distinct groups of proteins: helicases and chaperones. RNA helicases are highly similar to DNA helicases and use the energy provided by ATP hydrolysis to unwind RNA duplexes. These proteins are believed to participate in most ATP-dependent rearrangements of structured RNAs and are classified into six superfamilies (SFs) based on their conserved helicase motifs (Kadare and Haenni, 1997). Among these helicase superfamilies, SF1 and SF2 are the two largest and most closely related ones, and these SF1 and SF21 enzymes are monomeric and contain at least seven conserved motifs that mediate nucleic acid-binding, ATP-binding, ATP hydrolysis and conformational change of the helicases (Kadare and Haenni, 1997). Various RNA viruses have been found to encode their own RNA helicases, including brome mosaic virus (BMV) (Ahola et al., 2000; Wang et al., 2005), enterovirus (Mirzayan and Wimmer, 1992; Teterina et al., 1992; Xia et al., 2015), flavivirus (Gu et al., 2000), alphavirus (Rikkonen, 1996), alphatetravirus (Wang et al., 2012), and nidovirus (Oleksiewicz et al., 2000). 
Unlike RNA helicases, RNA chaperones are heterogeneous proteins that have no conserved motifs but are still able to destabilize or unwind RNA duplexes and promote the formation of correctly folded RNA structures by helping misfolded RNAs escape kinetic traps. RNA chaperones, however, do not require ATP binding or hydrolysis for RNA remodeling (Musier-Forsyth, 2010). The definition of RNA chaperones is somewhat uncertain, and some authors define RNA helicases as an ATP-dependent subgroup of RNA chaperones (Oleksiewicz et al., 2000). In this review, RNA helicases and RNA chaperones are defined as two separate classes of RNA remodelers.

Both RNA helicases and RNA chaperones function to destabilize or unwind RNA duplexes by binding to single-stranded RNA (ssRNA) strands. Their ssRNA binding and helicase/chaperone activities are not usually sequence-specific. Interestingly, another group of proteins, named ssRNA-binding proteins (sRBPs), are also able to destabilize RNA duplexes in an ATP-independent manner. The main difference between RNA chaperones and sRBPs, which stabilize a specific RNA conformation, is that the ssRNA binding by RNA chaperones is normally weak and transient, while sRBPs remain bound to ssRNAs to stabilize one strand and displace the other strand (Rajkowitsch et al., 2007; Zúñiga et al., 2009; Musier-Forsyth, 2010).

In viruses, particularly RNA viruses, the RNA molecules (vRNAs) can adopt various structures. They need proper structures to form diverse cis-acting elements in their 5'-untranslated region (UTR), 3'-UTR, or protein coding region; these elements are important for vRNA functions including translation, replication, and encapsidation (Rajkowitsch et al., 2007; Zúñiga et al., 2009; Musier-Forsyth, 2010). Formation of these highly structured RNA elements requires RNA chaperones to facilitate. The growing list of virus-encoded RNA chaperones currently includes the nucleocapsid (NC) protein of retroviruses, coronavirus nucleocapsid $(\mathrm{N})$ protein, hantavirus nucleocapsid $(\mathrm{N})$ protein, flavivirus core protein, enterovirus nonstructural proteins $3 \mathrm{AB}$ and $2 \mathrm{C}$, iflavirus $2 \mathrm{C}$, hepatitis D virus small delta antigen (SdAg), HIV-1 Tat and Vif, and cypovirus VP5. In addition, rotavirus nonstructural protein NSP2 is a putative RNA chaperone (Taraporewala and Patton, 2001). This review focuses on the RNA chaperones encoded by RNA viruses, including positive-stranded $(+)$, negative-stranded $(-)$, and double-stranded RNA viruses (Table 1).

\section{RNA CHAPERONES ENCODED BY (+)-RNA VIRUSES}

\section{Core proteins of Flaviviridae}

The viruses belonging to the family Flaviviridae have a single-stranded positive-sense RNA genome with a single open reading frame (ORF) which encodes a single polyprotein that is subsequently cleaved to produce individual structural and nonstructural proteins (Shepard et al., 2005). The family Flaviviridae comprises three genera, Flavivirus, Pestivirus and Hepacivirus (Shepard et al., 2005). The genus Hepacivirus includes hepatitis C virus (HCV), an important human pathogen causing chronic hepatitis and liver cirrhosis. The genus Flavivir$u s$ includes a variety of arthropod-borne viruses such as Dengue virus (DENV), yellow fever virus (YFV), West Nile virus (WNV), and others. The genus Pestivirus includes a number of important pathogens infecting livestock, such as classical swine fever virus (CSFV) and bovine viral diarrhea virus (BVDV).

The genomic RNA 5'UTR and 3'UTR of HCV contain a number of structured RNA elements that have been reported to be involved in viral replication (Ito and Lai, 1997; Friebe et al., 2005; Song et al., 2006). The HCV 5'-UTR contains 341 nucleotides (nt) and is folded into an internal ribosome entry site (IRES) structure that mediates cap-independent translation, while its 3 '-UTR is 230-nt long and contains three structured RNA elements, including a variable sequence with VSL1 and VSL2 stem-loops, a poly U/UC tract, and a 98-nt 3'X region that forms three stem-loops (Kolykhalov et al., 1996; Blight and Rice, 1997; Friebe and Bartenschlager, 2002). On cleavage, the HCV polyprotein yields three structural proteins, named core protein, E1 and E2. The core protein is located in the $\mathrm{N}$-terminal region of the polyprotein, contains many basic residues, and has RNAbinding activity. The HCV core protein may oligomerize upon packaging of the genomic RNA to form the viral nucleocapsid. The mature core protein has two domains, a hydrophobic $\mathrm{C}$-terminal domain and an N-terminal domain. The three very basic clusters in the N-terminal domain of $\mathrm{HCV}$ core protein have potent RNA chaperone activity that functions on the conserved $3^{\prime} \mathrm{X}$ region of the 3'-UTR of HCV genomic RNA and is able to direct the dimerization of HCV RNA 3'-UTRs (Kolykhalov et al., 2000; Boulant et al., 2006). Although the RNA chaperone activity of HCV core protein has been well characterized (Sharma et al., 2010), its functions in the life-cycle of HCV remain largely unknown.

Although RNA chaperones have been studied for many years, their ATP independent helix destabilizing and annealing mechanism has not been well understood. To explain the RNA chaperone activity, an "entropy transfer" model has been proposed in which the intrinsic disordered of chaperone proteins can transfer their entropy or disorder to RNA molecules. Such transfer of disorder destabilizes misfolded RNAs, helps them escape the trap of kinetic energy, and is conducive to the proper RNA structure reform. (Rajkowitsch et al., 2007; 
Table 1. The RNA chaperones encoded by RNA viruses.

\begin{tabular}{|c|c|c|c|c|c|}
\hline \multirow{9}{*}{$\begin{array}{l}\text { RNA } \\
\text { chaperone } \\
\text { encoded by } \\
(+)-R N A \\
\text { viruses }\end{array}$} & Family & Genus & Species & Protein Name & Reference \\
\hline & Flaviviridae & Hepacivirus & $\begin{array}{l}\text { Hepatitis } C \text { virus }(\mathrm{HCV}) \\
\text { GB virus } B(G B V-B)\end{array}$ & core protein & $\begin{array}{l}\text { Cristofari et al., 2004; } \\
\text { Ivanyi-Nagy et al., } 2006\end{array}$ \\
\hline & & Flavivirus & West niles virus & core protein & Ivanyi-Nagy et al., 2008 \\
\hline & & Pestivirus & $\begin{array}{l}\text { Bovine viral diarrhoea } \\
\text { virus (BVDV) }\end{array}$ & core protein & Ivanyi-Nagy et al., 2008 \\
\hline & Coronaviridae & Alphacoronavirus & $\begin{array}{l}\text { Transmissible } \\
\text { Gastroenteritis } \\
\text { virus (TGEV) }\end{array}$ & $\mathrm{N}$ protein & Zúñiga et al., 2007 \\
\hline & & Betacoronavirus & SARS-CoV & $\mathrm{N}$ protein & Cui et al., 2015 \\
\hline & Picornaviridae & Enterovirus & Poliovirus & $3 A B$ & $\begin{array}{l}\text { DeStefano and Titilope, } \\
2006\end{array}$ \\
\hline & & Enterovirus & Enterovirus 71 (EV71) & $3 A B, 2 C$ & $\begin{array}{l}\text { Tang et al., 2014; Xia et al., } \\
2015\end{array}$ \\
\hline & Iflaviridae & Iflavirus & $\begin{array}{l}\text { Ectropis obliqua picorna- } \\
\text { like virus (EoV) }\end{array}$ & $2 \mathrm{C}$ & Cheng et al., 2013 \\
\hline $\begin{array}{l}(-)-\text { RNA } \\
\text { virus }\end{array}$ & Bunyaviridae & Hantavirus & Sin Nombre hantavirus & $\begin{array}{l}\mathrm{N} \\
\text { nucleocapsid } \\
\text { protein }\end{array}$ & Mir and Panganiban, 2006 \\
\hline \multirow[t]{2}{*}{$\begin{array}{l}\text { dsRNA } \\
\text { viruses }\end{array}$} & Reoviridae & Cypovirus & Cypovirus 5 & VP5 & Yang et al., 2014 \\
\hline & & Rotavirus & Rotavirus & NSP2 & $\begin{array}{l}\text { Taraporewala and Patton, } \\
2001\end{array}$ \\
\hline
\end{tabular}

\section{Zúñiga et al., 2009).}

Interestingly, $\mathrm{HCV}$ core protein has been predicted to contain a disordered region, providing support to the "disorder or entropy transfer" model of RNA chaperones. Intrinsically disordered regions have also been predicted in the core proteins of other genera of Flaviviridae, including flavivirus and pestivirus. Biochemical analyses confirmed that these core proteins are also potent RNA chaperones (Ivanyi-Nagy et al., 2008).

\section{Coronavirus $\mathbf{N}$ protein}

Coronaviruses (CoVs) are enveloped viruses, belonging to the family Coronaviridae, including a large number of pathogens with global distribution that cause significant diseases in humans, such as Middle East respiratory syndrome (MERS) and severe acute respiratory syndrome (SARS) (Gorbalenya et al., 2006). The coronavirus genome is a single-stranded, positive-sense RNA, the largest viral RNA genome known (around 30 $\mathrm{kb}$ ), which is 5'-capped and 3'-polyadenylated (Sawicki and Sawicki, 2005). Six conserved genes are encoded, in the order 5'-ORF1a-ORF1b-S-E-M-N-3'. The 5' twothirds of the genomic RNA encode ORFs $1 \mathrm{a} / \mathrm{b}$, the viral replicase. The $3^{\prime}$ third of the genome encodes four structural proteins, membrane $(\mathrm{M})$, envelope $(\mathrm{E})$, spike $(\mathrm{S})$ and nucleocapsid $(\mathrm{N})$, and a number of nonstructural proteins scattered among them (Sawicki and Sawicki, 2005; Zúñiga et al., 2007).

Coronavirus $\mathrm{N}$ protein has a structural role in assembly of the virus, and is probably also involved in vir- al RNA synthesis (Zúñiga et al., 2007). Although different coronaviruses encode $\mathrm{N}$ proteins that vary in size and nucleotide sequence, the three-domain structure and some functional motifs are conserved, including a RNA binding domain, oligomerization domain, and $\mathrm{M}$ protein binding domain (Xia et al., 2015). Based on protein disorder analysis of coronavirus $\mathrm{N}$ proteins, RNA chaperone activity was first demonstrated for transmissible gastroenteritis virus (TGEV) N protein (Brian and Baric, 2005). RNA chaperone activity was also reported for SARS-CoV N protein, and it was suggested that RNA chaperone activity is a property of all coronavirus $\mathrm{N}$ proteins (Cui et al., 2015). Like other RNA chaperones, coronavirus $\mathrm{N}$ proteins also have activities such as facilitating nucleic acid annealing, RNA duplex destabilization, and enhancement of ribozyme self-cleavage (Cui et al., 2015).

TGEV N protein has RNA chaperone activity and is able to facilitate template switching in vitro (Zúñiga et al., 2007). Template switching during discontinuous RNA synthesis is a complicated and indispensable process that includes the stoppage of nucleic acid synthesis, the template switch, nascent nucleic acid strand re-association, and elongation of the nascent strand (Zúñiga et al., 2007). It is supposed that the $\mathrm{N}$ protein could decrease the thermodynamic barrier between the nascent $(-)$-RNA strand and the genomic RNA template, thereby promoting the nascent RNA strand template switch to hybridize with the transcription-regulating sequences of the leader sequence (Zúñiga et al., 2004). 


\section{Picornavirus $3 \mathrm{AB}$ protein}

Picornaviruses are a large group of single, plus-stranded RNA viruses, including many important human and animal pathogens, such as poliovirus (PV), foot-andmouth disease virus (FMDV), enterovirus 71 (EV71), and encephalomyocarditis virus (EMCV). Human picornaviruses cause over 3 billion human infections per year, and relating diseases ranging from mild (common cold, upper respiratory illness, and hand-foot-and-mouth disease), to severe (encephalitis, aseptic meningitis, neonatal sepsis-like disease, myocarditis, and poliomyelitis) conditions (Brian and Baric, 2005). The RNA genome of picornavirus is a single positive strand, containing a long 5'-UTR, a single ORF and a 3'-UTR. This RNA genome encodes a long polyprotein that is processed via cleavage at specific sites to produce mature viral proteins.

Protein $3 \mathrm{AB}$ is the precursor of nonstructural proteins $3 \mathrm{~A}$ and $3 \mathrm{~B}$. Protein $3 \mathrm{~A}$ is a membrane anchoring protein that anchors the replication complex to endoplasmic reticulum (ER) membrane (DeStefano and Titilope, 2006). Protein $3 \mathrm{~B}$, also called VPg, is a small peptide (21-24 amino acids (a.a.)) that is covalently linked to the 5 ' end of vRNAs and is believed function as a primer for initiating vRNA synthesis by the RNA-dependent RNA polymerase (RdRP), $3 \mathrm{D}^{\mathrm{Pol}}$. DeStefano and colleagues have showed that poliovirus protein $3 \mathrm{AB}$ possesses properties consistent with classical RNA chaperone proteins, and was able to greatly accelerate the rate of annealing between complementary nucleic acids and destabilize short double-stranded RNA substrates (DeStefano and Titilope, 2006). Moreover, our group has also demonstrated that protein $3 \mathrm{AB}$ from EV71 has RNA chaperone activity (Tang et al., 2014). These findings suggest that RNA chaperone activity is a general function of nonstructural protein $3 \mathrm{AB}$ in picornaviruses.

Poliovirus $3 \mathrm{AB}$ was the first RNA chaperone described in picornaviruses (DeStefano and Titilope, 2006). Domain organization and disorder prediction analyses showed that poliovirus or EV71 protein $3 \mathrm{AB}$ does not contain any intrinsically disordered regions, implying that the entropy transfer mechanism does not apply to enteroviral 3AB (Tang et al., 2014). However, the RNA chaperone activity of $3 \mathrm{AB}$ was moderately inhibited by various divalent metallic ions, implying that $3 \mathrm{AB}$ remodels RNA molecules through transient ionic interactions with the negatively charged RNA backbone (Woodson, 2010). The studies by Gangaramani et al. and Tang et al. have reported that the $3 \mathrm{~B}$ region plus the $\mathrm{C}$-terminal 7 a.a. of protein $3 \mathrm{~A}$ is the key region for the RNA chaperone activity of $3 \mathrm{AB}$ (Gangaramani et al., 2010; Tang et al., 2014). Moreover, residues Lys-81, Phe-83, His-86, Tyr-90 and Arg-104 of $3 \mathrm{AB}$ have been reported to be critical for the RNA chaperone activity of poliovirus or EV71 3AB (Gangaramani et al., 2010; Tang et al., 2014).
During the life cycle of picornaviruses, the RNA chaperone activity of $3 \mathrm{AB}$ may play critical roles in coating and protecting viral genomic RNA, facilitating the formation and/or deformation of vRNA cis-acting elements, vRNA synthesis, and translation. $3 \mathrm{AB}$ could strongly stimulate the RNA synthesis activity of $3 \mathrm{D}^{\mathrm{Pol}}$, especially when low concentrations of $3 \mathrm{D}^{\mathrm{Pol}}$ were used (Bystroff and Shao, 2002; James et al., 2003). However, for technical reasons, it is hard to determine the precise role of an RNA chaperone in a particular process in cells. Based on studies of protein $3 \mathrm{AB}$ from both poliovirus and EV71, several possibilities have been proposed, including: enhancement of primer binding to the template, facilitation and stabilization of picornaviral $3 \mathrm{D}^{\mathrm{Pol}}$ binding to the primer-template, and lowering of the dissociation rate constant (James et al., 2003; James et al., 2004).

\section{$2 C^{\text {ATPase }}$ proteins of picorna-like virus and picornavirus}

The picorna-like virus superfamily is a loosely defined group of positive-strand RNA viruses including 14 families of viruses and several unclassified genera and species. Among this superfamily, five families of viruses (Dicistroviridae, Iflaviridae, Marnaviridae, Picornaviridae and Secoviridae) make up the order Picornavirales. The genomes of picorna-like viruses are characterized by highly conserved genes, including an $\operatorname{RdRP}\left(a 3 \mathrm{D}^{\mathrm{Pol}}\right.$ for viral RNA replication), a chymotrypsin-like $3 C^{\text {Pro }}$ or $3 \mathrm{C}^{\text {Pro }}$-like (3CL) protease, and a putative helicase (nonstructural protein $\left.2 \mathrm{C}^{\mathrm{ATPase}}\right)$. Based on conserved superfamily 3 (SF3) signature A, B, and C motifs, $2 \mathrm{C}^{\text {ATPase }}$ has been predicted to be a SF3 helicase (Rodriguez and Carrasco, 1993; Pfister and Wimmer, 1999). Motifs A and B are "Walker" NTP binding motifs that appear in ATPand/or GTP-binding proteins (Rodriguez and Carrasco, 1993). Motif $C$ has been recognized as characteristic of SF3 helicases (Pfister and Wimmer, 1999). Although $2 \mathrm{C}^{\text {ATPase }}$ is one of the most conserved nonstructural proteins in the order Picornavirales, and multiple functional domains have been identified in genetic and drug inhibition studies, the exact roles of this protein in the viral life cycle remain largely unclear. Previous studies reported that $2 \mathrm{C}^{\text {ATPase }}$ participates in diverse processes, including RNA binding, RNA replication, inhibition of autophagy, suppression of nuclear factor-kappaB (NFkB) activation, membrane anchoring and rearrangement, and encapsidation and viral morphogenesis (Echeverri and Dasgupta, 1995; Teterina et al., 1997; Krogerus et al., 2003; Zheng et al., 2011; Gladue et al., 2012). Moreover, this protein appears to associate with vRNA in vRNA replication complexes in infected cells, implying a direct role in viral RNA replication (Rodriguez and Carrasco, 1995). 
$2 C^{\text {ATPase }}$ proteins of an insect picorna-like virus. RNA chaperone activity of a picorna-like virus $2^{\text {CATPase }}$ protein was first reported for the $2 \mathrm{C}^{\mathrm{ATPase}}$ from Ectropis obliqua picorna-like virus (EoV; genus Iflavirus, family Iflaviridae). The study by our group showed that EoV 2C ${ }^{\text {ATPase }}$ does have RNA remodeling activity that is able to destabilize RNA duplex. Surprisingly, the RNA remodeling activity associated with EoV $2 \mathrm{C}^{\text {ATPase }}$ is more like the activity of an RNA chaperone than the RNA helicase it was predicted to be (Cheng et al., 2013). EoV 2C $\mathrm{C}^{\text {ATPase }}$ can nonspecifically destabilize nucleic acid helices and accelerate strand annealing in an ATP-independent manner; and divalent metal ions (e.g. $\mathrm{Mg}^{2+}$ and $\mathrm{Zn}^{2+}$ ) inhibit $2 \mathrm{C}^{\text {ATPase }}$-mediated helix destabilization. EoV $2 \mathrm{C}^{\text {ATPase }}$ does have ATPase activity similar to that of other picornaviral $2 \mathrm{C}^{\mathrm{ATPase}}$ proteins, but the RNA chaperone and ATPase activities of EoV $2 \mathrm{C}^{\text {ATPase }}$ are independent and can be functionally separated (Cheng et al., 2013).

$2 C^{\text {ATPase }}$ proteins of picornaviruses. Enteroviruses are a large group of positive-stranded RNA viruses in the genus Enterovirus of the Picornaviridae family, and include many closely related human pathogens such as poliovirus, EV71, and coxsackie viruses. After the global eradication of poliovirus, EV71 is regarded as the most important neurotropic enterovirus. Recently, the highly conserved and multifunctional protein $2 \mathrm{C}^{\text {ATPase }}$ from EV71 has been demonstrated that functions as both an RNA helicase and an ATP-independent RNA chaperone, and that it is involved in viral RNA replication.

$2 \mathrm{C}^{\text {ATPase }}$ contains conserved motifs typical of SF3 helicases, but previous attempts to discover helicase activity associated with $2 \mathrm{C}^{\text {ATPase }}$ were unsuccessful (Rodriguez and Carrasco, 1993). A computation-modeled threedimensional structure of enteroviral $2 \mathrm{C}^{\text {ATPase }}$ indicated that this protein is similar to other viral SF3 helicases in terms of both conserved motifs and structure, implying that the protein should have RNA remodeling activity. Like other RNA remodeling proteins, EV71 2C ${ }^{\text {ATPase }}$ has helix unwinding activity for both RNA and DNA duplexes, and the unwinding activity is bidirectional. Moreover, destabilization of RNA duplexes by EV71 $2 \mathrm{C}^{\text {ATPase }}$ requires ATP or other NTPs. Interestingly, EV71 $2 \mathrm{C}^{\text {ATPase }}$ also has an ATP-independent helix unwinding activity. Furthermore, EV71 2C ${ }^{\text {ATPase }}$ unwinds 3'-protruded helices in an ATP-dependent manner, consistent with all known SF3 helicases, but the enzyme unwinds 5 '-protruded helices exactly like an RNA chaperone, i.e., in the absence of ATP (and such that increasing ATP concentrations did not further enhance the helix unwinding). Thus, one may speculate that EV71 2 $\mathrm{C}^{\text {ATPase }}$ has both RNA helicase and chaperone activities. The latter includes the ability to destabilize structured RNA strands, to stimulate RNA strand annealing, and to en- hance hammerhead ribozyme cleavage activity. Functional domain analysis showed that the C-terminal domain of $2 \mathrm{C}^{\text {ATPase }}$ plays an indispensable role in the RNA chaperone activity, probably due to the predicted coiledcoil structure that links the $\mathrm{C}$-terminal domain with the helicase core domain of $2 \mathrm{C}^{\mathrm{ATPase}}$ and the coiled-coil structure is intrinsically disordered. This finding also provides support for the "entropy or disorder transfer" model of RNA chaperones.

We proposed that during enteroviral RNA replication, particularly (+)-vRNA synthesis, $2 \mathrm{C}^{\text {ATPase }}$ initially associates with the $3^{\prime}$-end of the (-)-vRNA template, and its RNA chaperone activity functions to remodel and make the 3 '-end accessible to enteroviral RdRP $\left(3 \mathrm{D}^{\mathrm{Pol}}\right)$ and primers $(\mathrm{VPg})$, resulting in the initiation of $(+)$-vRNA replication (Figure 1, A-B). During the process of $(+)$ vRNA synthesis, the helicase activity of $2 \mathrm{C}^{\text {ATPase }}$ functions to unwind the replicative dsRNA, and even facilitates the release of 3DPol from the (-)-vRNA template, thereby releasing the nascently synthesized progeny $(+)$ vRNAs from the template (Figure 1, C-E), and recycling the (-)-stranded template and RdRP for another cycle of vRNA replication (Figure 1, E-A).

\section{RNA CHAPERONE ENCODED BY (-)-RNA VIRUS (BUNYAVIRUS N PROTEIN)}

Hantavirus, belonging to the family Bunyaviridae, is an enveloped, segmented, negative-stranded RNA virus (Khaiboullina et al., 2005). The virus genome includes three fragments, L, M and S, respectively encoding polymerase protein, G1 and G2 glycoproteins, and nuclear protein (Plyusnin et al., 1996; Barr and Wertz, 2005). There are two types of hantavirus, one causing hantavirus pulmonary syndrome (HPS), and the other causing hantavirus hemorrhagic fever with renal syndrome (HFRS). The former is mainly epidemic in the United States, and is also found in Argentina, Brazil, Paraguay, Bolivia and Germany. The latter is the common hemorrhagic fever with renal syndrome in China. It has been proven that the virus is the direct pathogen (Mei and Cosgriff, 1989). The kidney is the primary organ suffering injury in the early stage of infection, and the virus is the factor that directly causes renal damage.

Hantavirus $\mathrm{N}$ nucleocapsid protein plays an important role in the virus replication cycle. $\mathrm{N}$ protein has different activities and biological functions when interacting with, respectively, viral (-)RNA, $(+)$ cRNA, or mRNA. No mRNA was detected in encapsidation complex; the virus encapsidation process needs viral $(-)$ RNA or $(+) c$ RNA, but not mRNA.

The 5' and 3' ends of full-length the viral RNA of Bunyaviridae may base paired with each other and formed the intramolecular "panhandle" structures which 


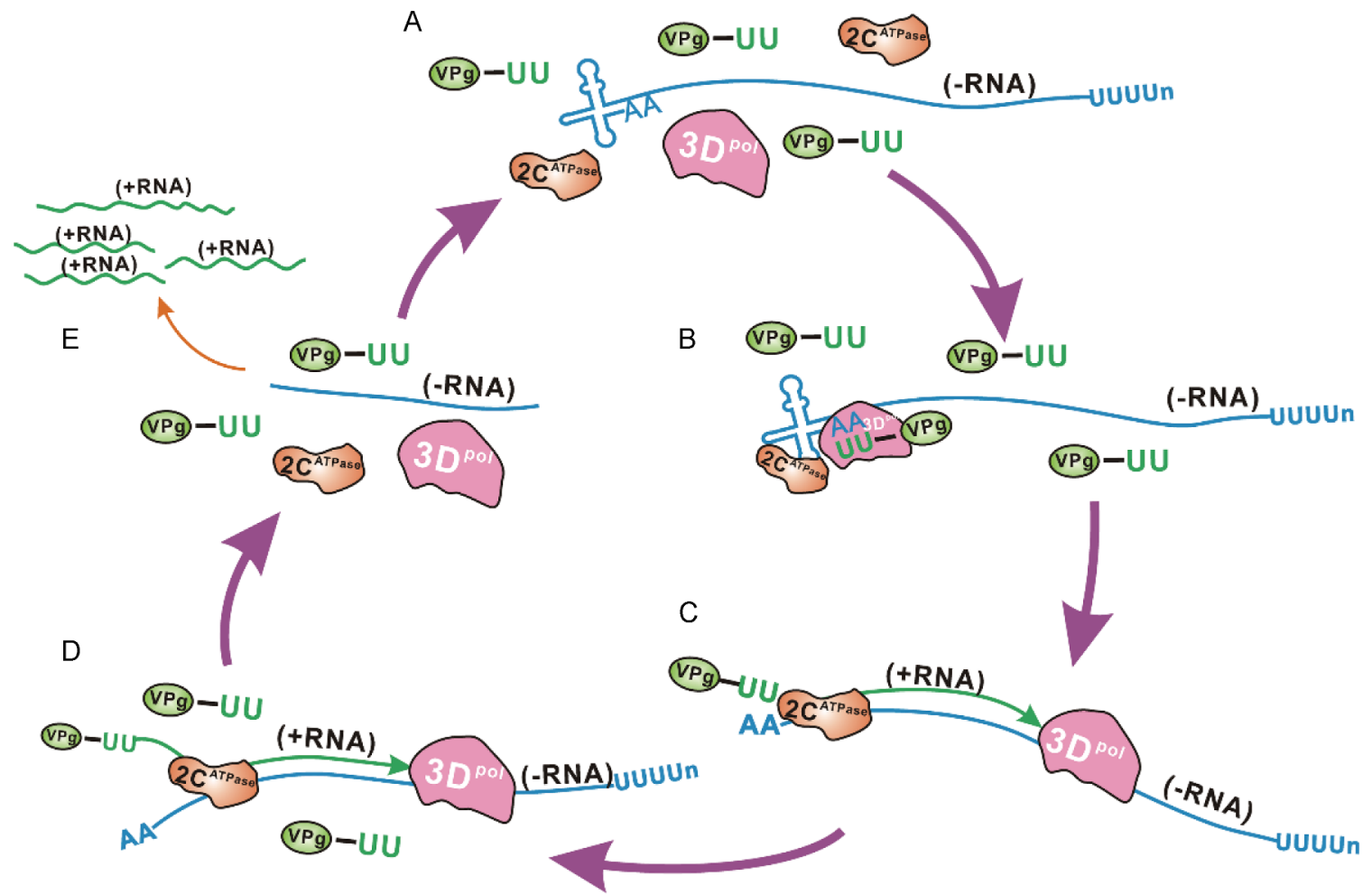

Figure 1. Model of $2 C^{\text {ATPase }}$ involvement in enteroviral replication. (A-B) Once enteroviral (-)RNA synthesis is complete, $3 \mathrm{D}^{\mathrm{pol}}$, the primer VPgpUpU, and other replication complex proteins are assembled at the 3 '-end of (-)-vRNA template, and then $3 D^{\text {pol }}$ initiates the synthesis of (+)-vRNA. In this step, the RNA chaperone activity of $2 C^{\text {ATPase }}$ functions to remodel and make the (-)-vRNA $3^{\prime}$-end accessible for $3 D^{\mathrm{Pol}}$ and the primers. (C-E) The process of (+)-vRNA synthesis on the (-)-vRNA template generates a replicative intermediate dsRNA region. $2 C^{\text {ATPase }}$ binds to the dsRNA (C) and its helicase activity functions to unwind the replicative dsRNA (D), resulting in the separation and release of nascently synthesized progeny (+)-vRNAs, and probably $3 D^{\text {Pol }}$ and VPgpUpU primers, from the (-)-vRNA template (E). (E-A) The (-)vRNA template, $3 \mathrm{D}^{\mathrm{Pol}}$, and VPgpUpU primers are then recycled for new rounds of vRNA replication.

is important for the recognition and encapsidation of vRNA during particle assembly (Mir and Panganiban, 2006). The RNA chaperone activity of $N$ protein can facilitate panhandle formation and is likely to be involved in initiation of translation (Mir and Panganiban, 2006). The N-terminal 100 amino acids of $\mathrm{N}$ protein form a domain that is both necessary and sufficient for the RNA chaperone activity. This region may be in one of two states: either highly disordered and functional in N-mediated RNA chaperone activity, or, in a trimeric form, ordered and functional in high-affinity vRNA panhandle recognition (Brown and Panganiban, 2010).

\section{RNA CHAPERONES ENCODED BY DSRNA VIRUSES}

\section{Cypovirus capsid protein VP5}

Reoviruses, belonging to the family Reoviridae, contain a segmented double-stranded RNA (dsRNA) gen- ome, and the genome was packaged in a single- or double-layered inner capsid. These viruses include important pathogens responsible for diseases in plants, insects, humans and livestock animals (Mertens, 2004). Unlike positive-stranded and negative-stranded RNA viruses, dsRNA viruses, including reoviruses, utilize their inner capsid not only as the shield for escaping antiviral defenses, but also as the machinery for vRNA synthesis, because the inner capsids of dsRNA viruses contain several copies of RdRPs and mRNA-capping enzyme (Mertens, 2004; Coulibaly et al., 2007).

Cypovirus (cytoplasmic polyhedrosis virus, CPV) is one of 15 genera within the family Reoviridae. Cypovirus contains 10 genomic dsRNA segments and is further classified into 16 types based on the electrophoretic migration profiles of the genome segments (King et al., 2011). CPV particles are packaged in polyhedra to enable survival in unfavorable external environments (Coulibaly et al., 2007). Cypovirus is used as a relatively simple model for studying dsRNA virus gene rep- 
lication because of its single layered capsid. The CPV capsid is comprised of three major capsid proteins, VP1, VP3, and VP5. One-hundred and twenty copies of VP1 form the capsid shell, 60 copies of VP3 are located on top of the five-fold axis and function as an mRNA capping enzyme, while 60 copies of VP5 are clamp proteins that stabilize the CPV capsid shell. It has recently been reported that VP5 from the type 5 Helicoverpa armigera cypovirus (HaCPV-5) possesses RNA chaperone activity and can destabilize RNA helices and accelerate strand annealing process ATP-independently (Yang et al., 2014). Further exploration of VP5 revealed that its helixdestabilizing activity is RNA-specific, lacks directional properties, and could be inhibited by divalent metal ions (e.g. $\mathrm{Mg}^{2+}, \mathrm{Mn}^{2+}, \mathrm{Ca}^{2+}$ and $\mathrm{Zn}^{2+}$ ) to varying degrees. The $5^{\prime}$ and $3^{\prime}$ ends of the (+)-vRNA strand contact and form a panhandle structure that has to be unlocked during genome replication. Moreover, it has been demonstrated that the VP5 protein of HaCPV-5 can facilitate the initiation of transcription through a CPV panhandle-structured RNA template by an alternative polymerase (i.e. a reverse transcriptase), which strongly suggests a direct function of the VP5 RNA chaperone activity in the initiation of cypoviral dsRNA replication (Yang et al., 2014).

\section{Rotavirus nonstructural protein 2 (NSP2)}

Rotavirus, another genus of the family Reoviridae, is the major cause of gastroenteritis in young children. It has been reported that the multifunctional enzyme NSP2 of rotavirus, involved in rotaviral dsRNA replication, has ATP-independent nucleic acid helix-destabilizing activity (Taraporewala and Patton, 2001). This activity lacks unwinding directionality and has no sequence specificity. The helix-destabilizing activity of RNA chaperones normally needs a large excess of protein over RNA helix substrate (DeStefano and Titilope, 2006; Cheng et al., 2013), and the optimal activity of rotavirus NSP2 has been detected at molar ratios of 1000:1 to 2000:1 (protein : RNA) (Taraporewala and Patton, 2001). These data suggest that CPV VP5 may not be the sole RNA chaperone protein in the family Reoviridae. However, rotavirus NSP2 and CPV VP5 have some differences. For example, CPV VP5 has RNA specific helix-destabilizing activity while NSP2 can unwind both DNA-RNA and RNA-RNA helices (Taraporewala et al., 1999; Taraporewala and Patton, 2001). This difference implies that these two reoviral proteins may use different mechanism to destabilize RNA helices. Since no study has yet determined whether rotavirus NSP2 possesses any strand annealing acceleration activity which is an important characteristic of RNA chaperones, further characterization of NSP2 is required to determine whether this protein should be recognized as an nucleic acid chaperone (Qiu et al., 2014).

\section{DISCUSSION}

Although the molecular mechanisms and structural bases governing ATP-dependent translocation and unwinding/destabilization by RNA helicases have been extensively studied (Kadare and Haenni, 1997; Rajkowitsch et al., 2007; Musier-Forsyth, 2010), the mechanism(s) by which RNA chaperones destabilize RNA duplexes and stimulate RNA strand annealing in an ATPindependent manner remain largely unknown. This discrepancy is partially due to the lack of consensus sequences or motifs among various RNA chaperones. Thus far, two hypotheses have been proposed. The most popular mechanistic hypothesis for RNA chaperone activity is the "disorder or entropy transfer" model, in which intrinsically disordered or unstructured regions of RNA chaperone proteins transfer their entropy (disorder) to the RNA molecules that they bind. This transfer of disorder destabilizes misfolded RNA base-pairings, helping them to escape kinetic traps and facilitating refolding to the correct RNA structure (Rajkowitsch et al., 2007; Zúñiga et al., 2009). Many virus-encoded RNA chaperone proteins, including HIV-1 Vif, NC, and Tat, flavivirus core, hantavirus $\mathrm{N}$ and coronavirus $\mathrm{N}$, are predicted to contain intrinsically disordered regions (Ivanyi-Nagy et al., 2008; Zúñiga et al., 2009; Brown and Panganiban, 2010), a prediction often made using the software DisProt Predictor VL3 (http://www.dabi.temple.edu/disprot/predictor.php) (Obradovic et al., 2003; Peng et al., 2005; Zúñiga et al., 2009). The disorder of hantavirus $\mathrm{N}$ was predicted using another algorithm, VL-XT (Brown and Panganiban, 2010); we reassessed it using VL3, and predict similar disordered regions. However, disorder prediction for poliovirus or EV71 3AB using VL3 suggests that these proteins do not contain disordered regions, and therefore that the "disorder or entropy transfer" model may not apply to all RNA chaperones.

Another model is that some RNA chaperones can remodel the RNA structures through transient electrostatic or ionic interactions with RNA molecules. For nucleic acids have negative charges on the phosphodiester backbone, ionic interactions may Seriously affect the dynamics and stability of RNA (Woodson, 2010). In this model, RNA chaperone proteins having a large, positively charged region could destabilize the structure of RNA and promote rearrangements of stable structure, and the cationic may inhibit strand annealing and RNA destabilization by stabilizing the RNA conformation and competing with RNA chaperones for non-specific RNA binding,. (Vo et al., 2006; Wu et al., 2007; Woodson, 2010). Because RNA chaperone proteins lack sequence similarity, it is highly likely that RNA chaperones employ diverse mechanisms for their functions. Future studies may elucidate these mechanisms. 
RNA chaperones and RNA helicases have been identified and studied extensively. However, the RNA remodeling activities of these proteins have normally been studied in vitro biochemically and biophysically; it is very hard to determine the cellular or in vivo roles of RNA helicase or chaperone activities on the structures and functionalities of RNAs. These technical limitations hinder our understanding of these important RNA-binding proteins and their roles in viral life cycles. Future studies may overcome these technical difficulties, providing a mechanistic view of how host or viral RNA remodeling proteins function in vivo.

\section{ACKNOWLEDGMENTS}

This work was supported by the National Basic Research Program of China (973 Program, 2014CB542603 to XZ), the National High-tech R\&D Program of China (863 Program, 2015AA020939 to XZ), the National Natural Science Foundation of China grants No. 31400141 (to JY) and No. 31270190 (to XZ), the Natural Science Foundation of Hubei grant No. 2015CFB351 (to JY), the National Science Foundation for Post-doctoral Scientists of China grant No. 2015M572190 (to JY).

\section{COMPLIANCE WITH ETHICS GUIDELINES}

The authors declare that they have no conflict of interest. This article does not contain any studies with human or animal subjects performed by any of the authors.

\section{REFERENCES}

Ahola T, den Boon JA, Ahlquist P. 2000. Helicase and capping enzyme active site mutations in brome mosaic virus protein 1a cause defects in template recruitment, negative-strand RNA synthesis, and viral RNA capping. J Virol, 74: 8803-8811.

Barr JN, Wertz GW. 2005. Role of the conserved nucleotide mismatch within 3'- and 5'-terminal regions of Bunyamwera virus in signaling transcription. J Virol, 79: 3586-3594.

Blight KJ, Rice CM. 1997. Secondary structure determination of the conserved 98-base sequence at the 3 ' terminus of hepatitis $\mathrm{C}$ virus genome RNA. J Virol, 71: 7345-7352.

Boulant S, Montserret R, Hope RG, Ratinier M, Targett-Adams P, Lavergne JP, Penin F, McLauchlan J. 2006. Structural determinants that target the hepatitis $\mathrm{C}$ virus core protein to lipid droplets. J Biol Chem, 281: 22236-22247.

Brian DA, Baric RS. 2005. Coronavirus genome structure and replication. Curr Top Microbiol Immunol, 287: 1-30.

Brown BA, Panganiban AT. 2010. Identification of a region of hantavirus nucleocapsid protein required for RNA chaperone activity. RNA Biol, 7: 830-837.

Bystroff C, Shao Y. 2002. Fully automated ab initio protein structure prediction using I-SITES, HMMSTR and ROSETTA. Bioinformatics, 18: S54-S61.

Chauhan S, Woodson SA. 2008. Tertiary interactions determine the accuracy of RNA folding. J Am Chem Soc, 130: 1296-1303.

Cheng Z, Yang J, Xia H, Qiu Y, Wang Z, Han Y, Xia X, Qin C-F,
Hu Y, Zhou X. 2013. The Nonstructural Protein 2C of a Picorna-like Virus Displays Nucleic Acid Helix Destabilizing Activity that can be Functionally Separated from its ATPase Activity. J Virol. 87:5205-5218.

Coulibaly F, Chiu E, Ikeda K, Gutmann S, Haebel PW, SchulzeBriese C, Mori H, Metcalf P. 2007. The molecular organization of cypovirus polyhedra. Nature, 446: 97-101.

Cui L, Wang H, Ji Y, Yang J, Xu S, Huang X, Wang Z, Qin L, Tien P, Zhou X, Guo D, Chen Y. 2015. The Nucleocapsid Protein of Coronaviruses Acts as a Viral Suppressor of RNA Silencing in Mammalian Cells. J Virol, 89: 9029-9043.

DeStefano JJ, Titilope O. 2006. Poliovirus Protein 3AB Displays Nucleic Acid Chaperone and Helix-Destabilizing Activities. J Virol, 80: 1662-1671.

Echeverri AC, Dasgupta A. 1995. Amino terminal regions of poliovirus $2 \mathrm{C}$ protein mediate membrane binding. Virology, 208: 540-553.

Friebe P, Bartenschlager R. 2002. Genetic analysis of sequences in the 3 ' nontranslated region of hepatitis $\mathrm{C}$ virus that are important for RNA replication. J Virol, 76: 5326-5338.

Friebe P, Boudet J, Simorre JP, Bartenschlager R. 2005. Kissingloop interaction in the $3^{\prime}$ end of the hepatitis $\mathrm{C}$ virus genome essential for RNA replication. J Virol, 79: 380-392.

Gangaramani DR, Eden EL, Shah M, Destefano JJ. 2010. The twenty-nine amino acid C-terminal cytoplasmic domain of poliovirus $3 \mathrm{AB}$ is critical for nucleic acid chaperone activity. RNA Biol, 7: 820-829.

Gladue DP, O'Donnell V, Baker-Branstetter R, Holinka LG, Pacheco JM, Fernandez-Sainz I, Lu Z, Brocchi E, Baxt B, Piccone ME, Rodriguez L, Borca MV. 2012. Foot-and-mouth disease virus nonstructural protein $2 \mathrm{C}$ interacts with Beclin1, modulating virus replication. J Virol, 86: 12080-12090.

Gorbalenya AE, Enjuanes L, Ziebuhr J, Snijder EJ. 2006. Nidovirales: evolving the largest RNA virus genome. Virus Res, 117: 17-37.

Gu B, Liu C, Lin-Goerke J, Maley DR, Gutshall LL, Feltenberger CA, Del Vecchio AM. 2000. The RNA helicase and nucleotide triphosphatase activities of the bovine viral diarrhea virus NS3 protein are essential for viral replication. J Virol, 74: 1794-1800.

Ito T, Lai MM. 1997. Determination of the secondary structure of and cellular protein binding to the 3'-untranslated region of the hepatitis C virus RNA genome. J Virol, 71: 8698-8706.

Ivanyi-Nagy R, Lavergne JP, Gabus C, Ficheux D, Darlix JL. 2008. RNA chaperoning and intrinsic disorder in the core proteins of Flaviviridae. Nucleic Acids Res, 36: 712-725.

James JA, Aggarwal AK, Linden RM, Escalante CR. 2004. Structure of adeno-associated virus type 2 Rep40-ADP complex: insight into nucleotide recognition and catalysis by superfamily 3 helicases. Proc Natl Acad Sci U S A, 101: 12455-12460.

James JA, Escalante CR, Yoon-Robarts M, Edwards TA, Linden RM, Aggarwal AK. 2003. Crystal structure of the SF3 helicase from adeno-associated virus type 2. Structure, 11: 1025-1035.

Kadare G, Haenni AL. 1997. Virus-encoded RNA helicases. J Virol, 71: 2583-2590.

Khaiboullina SF, Morzunov SP, St Jeor SC. 2005. Hantaviruses: molecular biology, evolution and pathogenesis. Curr Mol Med, 5: 773-790.

King AM, Adams MJ, Lefkowitz E. 2011. Virus taxonomy: ninth report of the International Committee on Taxonomy of Viruses, vol. 9. Elsevier.

Kolykhalov AA, Feinstone SM, Rice CM. 1996. Identification of a highly conserved sequence element at the 3 ' terminus of hepatitis C virus genome RNA. J Virol, 70: 3363-3371.

Kolykhalov AA, Mihalik K, Feinstone SM, Rice CM. 2000. Hepatitis $\mathrm{C}$ virus-encoded enzymatic activities and conserved RNA elements in the 3' nontranslated region are essential for virus replication in vivo. J Virol, 74: 2046-2051.

Krogerus C, Egger D, Samuilova O, Hyypia T, Bienz K. 2003. 
Replication complex of human parechovirus 1. J Virol, 77: 8512-8523.

Mei YG, Cosgriff TM. 1989. Hemorrhage in hemorrhagic fever with renal syndrome in China. Reviews of Infectious Diseases, 11: S884-S890.

Mertens P. 2004. The dsRNA viruses. Virus Research, 101: 3-13.

Mir MA, Panganiban AT. 2006. The bunyavirus nucleocapsid protein is an RNA chaperone: possible roles in viral RNA panhandle formation and genome replication. Rna, 12: 272-282.

Mirzayan C, Wimmer E. 1992. Genetic analysis of an NTP-binding motif in poliovirus polypeptide 2C. Virology, 189: 547-555.

Musier-Forsyth K. 2010. RNA remodeling by chaperones and helicases. RNA Biol, 7: 632-633.

Obradovic Z, Peng K, Vucetic S, Radivojac P, Brown CJ, Dunker AK. 2003. Predicting intrinsic disorder from amino acid sequence. Proteins, 53 Suppl 6: 566-572.

Oleksiewicz MB, Botner A, Toft P, Grubbe T, Nielsen J, Kamstrup S, Storgaard T. 2000. Emergence of porcine reproductive and respiratory syndrome virus deletion mutants: correlation with the porcine antibody response to a hypervariable site in the ORF 3 structural glycoprotein. Virology, 267: 135-140.

Peng K, Vucetic S, Radivojac P, Brown CJ, Dunker AK, Obradovic Z. 2005. Optimizing long intrinsic disorder predictors with protein evolutionary information. J Bioinform Comput Biol, 3: 35-60.

Pfister T, Wimmer E. 1999. Characterization of the nucleoside triphosphatase activity of poliovirus protein $2 \mathrm{C}$ reveals a mechanism by which guanidine inhibits poliovirus replication. J Biol Chem, 274: 6992-7001.

Plyusnin A, Vapalahti O, Vaheri A. 1996. Hantaviruses: genome structure, expression and evolution. J Gen Virol, 77: 26772687.

Qiu Y, Miao M, Wang Z, Liu Y, Yang J, Xia H, Li XF, Qin CF, $\mathrm{Hu}$ Y, Zhou X. 2014. The RNA binding of protein A from Wuhan nodavirus is mediated by mitochondrial membrane lipids. Virology, 462-463: 1-13.

Rajkowitsch L, Chen D, Stampfl S, Semrad K, Waldsich C, Mayer O, Jantsch MF, Konrat R, Blasi U, Schroeder R. 2007. RNA chaperones, RNA annealers and RNA helicases. RNA Biol, 4: $118-130$

Rikkonen M. 1996. Functional significance of the nuclear-targeting and NTP-binding motifs of Semliki Forest virus nonstructural protein nsP2. Virology, 218: 352-361.

Rodriguez PL, Carrasco L. 1993. Poliovirus protein 2C has ATPase and GTPase activities. J Biol Chem, 268: 8105-8110.

Rodriguez PL, Carrasco L. 1995. Poliovirus protein 2C contains two regions involved in RNA binding activity. J Biol Chem, 270: 10105-10112.

Sawicki SG, Sawicki DL. 2005. Coronavirus transcription: a perspective. Curr Top Microbiol Immunol, 287: 31-55.

Sharma K, Didier P, Darlix JL, de Rocquigny H, Bensikaddour H, Lavergne JP, Penin F, Lessinger JM, Mely Y. 2010. Kinetic analysis of the nucleic acid chaperone activity of the hepatitis $\mathrm{C}$ virus core protein. Nucleic Acids Res, 38: 3632-3642.

Shepard CW, Finelli L, Alter MJ. 2005. Global epidemiology of hepatitis $C$ virus infection. Lancet Infect Dis, 5: 558-567.

Song Y, Friebe P, Tzima E, Junemann C, Bartenschlager R, Niepmann M. 2006. The hepatitis C virus RNA 3'-untranslated region strongly enhances translation directed by the internal ribo- some entry site. J Virol, 80: 11579-11588.

Tang F, Xia H, Wang P, Yang J, Zhao T, Zhang Q, Hu Y, Zhou X. 2014. The identification and characterization of nucleic acid chaperone activity of human enterovirus 71 nonstructural protein 3AB. Virology, 464-465: 353-364.

Taraporewala Z, Chen D, Patton JT. 1999. Multimers formed by the rotavirus nonstructural protein NSP2 bind to RNA and have nucleoside triphosphatase activity. J Virol, 73: 9934-9943.

Taraporewala ZF, Patton JT. 2001. Identification and Characterization of the Helix-Destabilizing Activity of Rotavirus Nonstructural Protein NSP2. J Virol, 75: 4519-4527.

Teterina NL, Kean KM, Gorbalenya AE, Agol VI, Girard M. 1992. Analysis of the functional significance of amino acid residues in the putative NTP-binding pattern of the poliovirus 2C protein. J Gen Virol, 73: 1977-1986.

Teterina NL, Gorbalenya AE, Egger D, Bienz K, Ehrenfeld E. 1997. Poliovirus 2C protein determinants of membrane binding and rearrangements in mammalian cells. J Virol, 71: 89628972.

Vo MN, Barany G, Rouzina I, Musier-Forsyth K. 2006. Mechanistic studies of mini-TAR RNA/DNA annealing in the absence and presence of HIV-1 nucleocapsid protein. J Mol Biol, 363: 244-261.

Wang Q, Han Y, Qiu Y, Zhang S, Tang F, Wang Y, Zhang J, Hu Y, Zhou X. 2012. Identification and characterization of RNA duplex unwinding and ATPase activities of an alphatetravirus superfamily 1 helicase. Virology, 433: 440-448.

Wang X, Lee WM, Watanabe T, Schwartz M, Janda M, Ahlquist P. 2005. Brome mosaic virus la nucleoside triphosphatase/helicase domain plays crucial roles in recruiting RNA replication templates. J Virol, 79: 13747-13758.

Woodson SA. 2010. Taming free energy landscapes with RNA chaperones. RNA Biol, 7: 677-686.

Wu T, Heilman-Miller SL, Levin JG. 2007. Effects of nucleic acid local structure and magnesium ions on minus-strand transfer mediated by the nucleic acid chaperone activity of HIV-1 nucleocapsid protein. Nucleic Acids Res, 35: 3974-3987.

Xia H, Wang P, Wang GC, Yang J, Sun X, Wu W, Qiu Y, Shu T, Zhao X, Yin L, Qin CF, Hu Y, Zhou X. 2015. Human Enterovirus Nonstructural Protein 2CATPase Functions as Both an RNA Helicase and ATP-Independent RNA Chaperone. PLoS Pathog, 11: e1005067.

Yang J, Cheng Z, Zhang S, Xiong W, Xia H, Qiu Y, Wang Z, Wu F, Qin CF, Yin L, Hu Y, Zhou X. 2014. A cypovirus VP5 displays the RNA chaperone-like activity that destabilizes RNA helices and accelerates strand annealing. Nucleic Acids Res, 42: $2538-2554$

Zheng Z, Li H, Zhang Z, Meng J, Mao D, Bai B, Lu B, Mao P, Hu Q, Wang H. 2011. Enterovirus $712 \mathrm{C}$ protein inhibits TNF-alpha-mediated activation of NF-kappaB by suppressing IkappaB kinase beta phosphorylation. J Immunol, 187: 2202-2212.

Zúñiga S, Sola I, Alonso S, Enjuanes L. 2004. Sequence motifs involved in the regulation of discontinuous coronavirus subgenomic RNA synthesis. J Virol, 78: 980-994.

Zúñiga S, Sola I, Cruz JL, Enjuanes L. 2009. Role of RNA chaperones in virus replication. Virus Res, 139: 253-266.

Zúñiga S, Sola I, Moreno JL, Sabella P, Plana-Durán J, Enjuanes L. 2007. Coronavirus nucleocapsid protein is an RNA chaperone. Virology, 357: 215-227. 